Psychological Medicine, 1982, 12, 225-230

Printed in Great Britain

\title{
EDITORIAL
}

\section{Interhemispheric integration in man ${ }^{1}$}

Twenty years ago a group of distinguished scientists - physiologists, neurologists, anatomists, psychologists and linguists - met at the Johns Hopkins School of Medicine to discuss the functional relationships between the two cerebral hemispheres. Their discussion centred around the simple question 'Why have we two brains?', and details of the proceedings of this conference are contained in a monograph entitled Interhemispheric Relations and Cerebral Dominance (Mountcastle, 1962). In summing up these proceedings, it was pointed out that the lack of any clear understanding of the ways in which brain commisures act to provide a transfer of information between the two cerebral hemispheres forms a major gap in the field of neuroscience.

Ten years later, in 1971, another group of eminent research workers gathered for the third study programme in the neurosciences at Colorado (Schmitt \& Worden, 1974). At this meeting Teuber (1974) presented a paper entitled 'Why two brains?', in which he stressed the limitations of existing knowledge concerning the fundamental question of 'How commisures act in providing information transfer between the hemispheres and in constraining, or modulating, the activities of the parallel halves of the brain in such a way that a functional asymmetry arises and is maintained'.

Today the question of how interaction between the two hemispheres is obtained still remains unanswered.

This gap in our knowledge might be at least partly explained by the almost exclusive interest, over recent years, in establishing differential specialization of the right and left hemispheres in the normal brain (see Dimond, 1972; Kinsbourne, 1978; Bradshaw \& Nettleton, 1981). To a great extent this is a consequence of techniques becoming available which permit a behavioural analysis of the two halves of the intact brain. Previously, the only way to obtain information about the dual functional asymmetry of the human brain had been by experimental investigations carried out on patients with unilateral brain lesions (see Hécaen, 1962; Zangwill, 1963). The studies of normal individuals were designed in an attempt, not only to determine those crucial aspects of cognitive function which distinguish right and left hemispheres, but also to demonstrate differences that exist between different groups of individuals - for example, between men and women (see Buffery \& Gray, 1972; Harshman \& Remington, 1975), children and adults (see Harnard et al. 1977; Dimond \& Blizard, 1977), right and left handers (see Hécaen \& Ajuriaguerra, 1964; Corballis \& Beale, 1976). These studies have taught us a great deal and, as a result, the concept of left cerebral dominance has been abandoned, to be replaced by one of complementary specialization. For some time the roles of the left and the right cerebral hemispheres were described in terms of a verbal/non-verbal dichotomy, with the left hemisphere dominant for language functions and the right for visuo-spatial abilities. This view, however, has now been challenged, and the roles of the hemispheres have been interpreted on an entirely different basis (see Bradshaw \& Nettleton, 1981). For instance, the left hemisphere is no longer described as possessing a specialized mechanism for the processing of speech; rather, its function is explained as having a unique specialization for the organization of temporal order sequencing and segmentation. Although the question of what, precisely, are the special functions of the two hemispheres remains open, the fact of a dual functional asymmetry of the two halves of the brain is now generally accepted.

At present, however, it is felt that too much time has been devoted to separating the functions of the respective cerebral hemispheres and too little to analysing how they interact. And it might, at this point, be instructive to bear in mind that between the 'two brains' there is one of the most

\footnotetext{
1 This paper is based on a talk given at the Institute of Psychiatry, on 13 February 1980. Address for correspondence: Dr Maria Wyke, Department of Psychology, Institute of Psychiatry, De Crespigny Park, Denmark Hill, London SE5 8AF.
} 
extensive bands of fibres in the nervous system: the corpus callosum, which must serve some other purpose than simply carrying a one-way traffic of information, or as has been stated in the past 'to aid the transmission of epileptic seizures from one side of the body to the other', or, just to perform a simple mechanical role 'to keep the hemispheres from sagging' (see Sperry, 1962). It is now time to turn our attention to establishing how the two distinct and dissimilar brains interact in order to bring about the organized and integrated behaviour of normal individuals.

Others have already drawn attention to the need to solve this problem, among them Broadbent (1974) who, in the same study programme of neurosciences in 1972, argued convincingly for the need to see the cerebral hemispheres as 'performing different parts of an integrated performance, rather than completely separate and parallel functions'.

This paper presents behavioural evidence in support of the notion that the functional specialization of the two hemispheres does not necessarily imply a parallel function or total independence, but that their functions do, in fact, interact. Much of this evidence for interaction between the two cerebral hemispheres has been derived from the very same techniques used to study cerebral. specialization, and a brief description of these techniques will follow. They have involved comparing differences in performance between the right and left side of the visual field, by analysing responses to stimuli presented to the left and right ear, and also assessing the performance of voluntary movements with the right and left arms.

The anatomical and physiological arrangement of the visual system - with stimuli presented to the left visual field being processed by the right hemisphere and vice versa - provides us with the opportunity to study effectively the different modes of action of the two hemispheres. The possible interference of eye movements can be eliminated by making the presentation brief - in milliseconds. The accuracy and speed with which these presentations are reported has been taken as evidence of cerebral specialization. For example, when printed words are presented to the left and right of the fixation points and the subject reports more accurately those shown in the right field, this has been taken to imply that the left hemisphere is superior, or dominant, in the processing of verbal material. Similarly, evidence of hemispheric specialization in the auditory modality has been obtained by using the dichotic listening technique. This involves the simultaneous presentation of different messages to the two ears. Greater cortical responses to stimulation of the contralateral ear than to stimulation of the ipsilateral ear reveal the asymmetry of function, and this effect is accentuated when stimulation is simultaneous and competitive (Rosenzweig, 1951). For example, when a different set of words is simultaneously presented to each ear and there is greater accuracy of identification in the right ear, this is taken as evidence that the left hemisphere is dominant in the perception of verbal material (see Milner, 1971; Hécaen \& Albert, 1978).

The neural organization of the motor system is somewhat similar to that of the auditory system. The left hemisphere controls mainly the somatosensory and motor functions of the right limbs, while the right hemisphere controls the functions of the left arm and leg. Separation is not complete, as there is ample physiological and anatomical evidence for the existence of an ipsilateral control of sensory-motor function (Terzulo \& Adey, 1960; Patton et al. 1962; Goff et al. 1962).

Evidence for cerebral specialization can be, and frequently is, taken too literally - that is, it is taken to imply that the two halves of the normal brain perform separate and parallel functions. There is, however, substantial evidence of interaction which may suggest that integration of the activity of the two hemispheres is, in fact, the normal and customary mode of function of the intact brain. The term 'interaction' is used here to mean 'mutual and reciprocal' influence between the two halves of the brain. This definition goes well beyond that which describes a simple one-way traffic of information and attributes a role to the cerebral commissures which is much more than merely informing one hemisphere of the activities of the other. Rather, the commissures' function is that of exerting reciprocal influence between the two hemispheres. There are a number of ways in which we can demonstrate the presence of such interaction. For instance, by showing the existence of cooperative activity between the hemispheres - that is to say: an increased proficiency at performing a task when the two hemispheres are involved; and by demonstrating interference - that is, when one hemisphere interferes with, or hinders, the activity of the other. It could also be demonstrated by 
showing transfer of learning - for example, when activity learned by one hemisphere is transferred to the other; by showing inhibition - that is, when one hemisphere inhibits or takes control of the latent activity of the other; and by showing suppression - that is, when one hemisphere suppresses the outgoing activity of the other.

Evidence for interhemispheric interaction comes from various behavioural studies. The first example of cooperative activity comes from a study made by Dimond \& Beaumont (1972). They presented, tachistoscopically, simple non-verbal stimuli to a group of normal subjects. The stimulus material consisted of pairs of semicircles with various symmetrical designs. The subjects were shown two of these designs in succession and were simply required to identify whether the first and second designs were the same. There were three different modes of presentation of the stimuli to the right visual field, to the left visual field, and to both fields.

There were two different means of response: vocal and manual, the latter requiring the subject to depress two micro switches, one held in each hand. On the basis of existing information it might be assumed that, since this was a non-verbal task, the right hemisphere would be superior in processing this type of information. This would mean that the responses to stimuli presented to the left visual field alone would be significantly faster. In fact, this was not the case. The fastest responses actually occurred when the stimuli were presented to both visual fields: that is, when the two hemispheres were involved in the decision. Dimond \& Beaumont interpreted their findings as showing that when the perceptual load was divided between the two hemispheres, efficiency was accentuated.

Another experimental study demonstrating cooperative interaction comes from Basso et al. (1977). They based their study on the well known perceptual experience of brightness contrast (see Forgus, 1966). It has been shown that if a shade of grey is surrounded by a white background it appears darker than if it is surrounded by a black background. Basso et al. used cards with areas of grey half surrounded by a white background and the other half by a black background. Their subjects were asked to fixate their eyes on the middle of the card in such a way that half the card (for instance, the grey area with white background) was processed by one hemisphere while the other half (in this case the grey with the black background) was simultaneously processed by the other hemisphere. The subjects were then asked to match the grey that they perceived with one on a multiple choice display. This experimental condition, with a different perception of the greyness reaching each hemisphere, provided a perceptual ambiguity. Decision regarding the greyness of the area would, according to existing information, be determined by the right hemisphere which is dominant for the perception of non-verbal material. Thus it was predicted that the subjects would opt for the grey which matched that presented in their left visual field. The results, however, showed that the subjects' responses were an averaging of the information available to each hemisphere. In other words, the grey selected was neither of the two stimuli processed by the respective hemispheres, but rather the decision was based on the information derived from both. Thus it clearly demonstrated a case of mutual cooperation between the two halves of the brain.

As has been mentioned above, if the activity of one hemisphere is seen to interfere with the activity of the other this may be taken as evidence of inter-hemispheric interaction, as the two following studies show precisely. The first comes from Broadbent \& Gregory (1965) and Broadbent (1974). These authors argued that, if the two cerebral hemispheres were indeed two parallel channels of communication, then it ought to be possible for a normal subject to perform two different activities simultaneously: one which is known to be subserved by the left hemisphere, the other by the right. To test this notion they studied the reaction time to the stimulation of the finger tip of the left hand. The stimulation occurred every five seconds and the subjects were required to depress a key with the finger stimulated. At the same time they were receiving, every five seconds, a spoken letter of the alphabet. These letters were normally different, but within each minute one letter occurred twice. The subjects were required to report verbally, each minute, which letter appeared most frequently. Thus the subject was simultaneously performing a manual reaction to touch and a spoken reaction based upon memory of speech stimuli - with the right hemisphere controlling the somatosensory and motor performances of the left hand and the left hemisphere, dominant for the language 
function, subserving the verbal task. This experimental situation would allow the two halves of the brain to work simultaneously in an independent and parallel fashion. Broadbent \& Gregory, however, found that when the two stimuli coincided the subject had both a diminished memory for the spoken letters and a significantly slower reaction time to the finger stimulation. The implication of these results is that, since the two hemispheres interfere with each other's action, they cannot be working independently or in parallel; rather, they must be interacting.

It could be argued, however, that the tasks which Broadbent \& Gregory required their subjects to perform were too complex, and that the interference arose because their subjects had to divide their attention. But this argument cannot be maintained if interference can be demonstrated when the subject is performing simple, identical, repetitive tasks. Wyke (1969) studied the ability of subjects to perform simple tapping movements with the two hands. The subject's task was simply to tap on a board with a stylus in an up-and-down movement. All the subjects were tested using the right and left hands independently and also using both hands simultaneously. Tapping with the right hand (all the subjects were right-handed) was invariably faster than tapping with the left hand, but when both hands were tapping simultaneously the speed dropped to a level which was significantly lower than for the left hand alone. So, even when they are performing simple repetitive tasks, the arms cannot be regarded as independent moving parts controlled, in parallel, by the two cerebral hemispheres. There is interference, even at this level.

The point concerning the transfer of learning between the two hemispheres is demonstrated by looking at an example of negative transfer. Heap \& Wyke (1972) studied the learning of a unimanual motor skill in normal subjects. They used a target pursuit rotor apparatus, consisting of an illuminated target which moves in a circular manner around the glass top of the apparatus, and asked their subjects to follow the target with the probe using one hand. Accuracy of performance was assessed by measuring the total length of time that the subject managed to keep the probe in contact with the target.

Subjects attempted 10 trials of fifteen seconds, each using one hand followed immediately by the same number of trials using the other hand. Subjects were sub-divided into two groups: those who started the test with the right arm and followed with the left, and those who carried out the task in reverse sequence. Results showed that the efficiency of the right arm at performing the task significantly decreased when it was used second in the testing sequence. A decrease in performance was also recorded when the left arm was used second in the sequence, but here the difference was not statistically significant. So it can be said that decreased efficiency of performance with the right arm was a case of negative transfer of learning, although it is of interest that this negative transfer of learning only occurs when the left hand is trained first. The findings, however, reinforce the view that the arms are not independent units controlled in a simple manner by the contralateral hemisphere.

Finally, the information obtained on the two areas of hemispheric interactions mentioned above - the control, by one hemisphere, of the latent activity of the other and the suppression, by one hemisphere, of the outgoing activities of the other - has been derived from the clinical field. Several workers (see von Békésy, 1967) have provided evidence for inhibitory control of one hemisphere over the other. One instance refers to the inhibition of speech function. It has been argued that the right hemisphere possesses a limited ability for verbal expression (Gazzaniga \& Hillyard, 1971). This ability, however, is restricted by the left hemisphere's predominance in the control of the neuro-muscular mechanisms involved in speech (Butler \& Norrsell, 1968; Levy et al. 1971). Release of the inhibition of speech mechanisms by the left hemisphere has been demonstrated in the case of some aphasic patients. Kinsbourne (1971) studied three right-handed patients who had suffered left hemisphere strokes which caused aphasia. All three subjects had some residual speech functions. Injection of sodium amylobarbitone into the right carotid circulation caused a complete arrest of speech, while this was not the case when the injection was on the left. These observations were interpreted by claiming that whatever speech these patients retained was subserved by the right and not by the left hemisphere. This implies that only when the left hemisphere relinquishes control of the neuro-muscular mechanisms can the right hemisphere 'speak'. In other words, the right 
hemisphere is capable of speech production and can, in fact, subserve oral language when, as a result of brain damage, the left hemisphere surrenders control of the peripheral apparatus of speech production.

Analysis of voluntary movements can also demonstrate a similar suppression of outgoing activity. It is well known that in young children voluntary movements of one limb, and especially the hand, are frequently accompanied by contralateral movements (Fog \& Fog, 1963; Abercrombie et al. 1964). The natural tendency is for these movements to disappear after the first decade (Fog \& Fog, 1963). Their suppression, however, is not total as it has been demonstrated, by means of electromyography, that during voluntary movements of one arm there is contraction of the symmetrical muscles on the opposite side (Cernacek, 1961; Hopf et al. 1974). Occasionally, diseases can damage these suppression mechanisms (see Schott \& Wyke, 1977, 1981) and give rise to abnormal synkinesias. There are also rare cases in which the mechanism fails to develop altogether, producing abnormal obligatory movements in otherwise normal subjects (Schott \& Wyke, 1977). The conclusion we can draw from the suppression of this contralateral motor activity is that there is a constant interaction between the two cerebral hemispheres.

At present, the mechanisms of interaction between the two hemispheres are poorly understood. Moreover, theoretical models which might explain the different aspects of this interaction are lacking. A great deal of research remains to be done. We may at least hope that, in the future, the same zeal which has been applied to separating the activities of the two cerebral hemispheres can be applied to unifying them.

MARIA WYKE

\section{REFERENCES}

Abercrombie, M. L., Lindon, R. L. \& Tyson, M. C. (1964). Associated movements in normal and physically handicapped children. Developmental Medicine and Child Neurology 6, 573580.

Basso, A., Bisiach, E. \& Capitani, E. (1977). Decision in ambiguity: hemispheric dominance or interaction? Cortex 13, 96-99.

Békésy, G. von (1967). Sensory Inhibition. Princeton University Press: Princeton, N.J.

Bradshaw, J. L. \& Nettleton, N. C. (1981). The nature of hemisphere specialization in man. Behavioural and Brain Sciences 4, 51-91.

Broadbent, D. E. (1974). Division of function and integration of behaviour. In The Neurosciences Third Study Program (ed. F. O. Schmitt and F. G. Worden), pp. 31-41. MIT Press: Cambridge, Mass.

Broadbent, D. E. \& Gregory, M. (1965). On the interaction of S-R compatibility with other variables affecting reaction time. British Journal of Psychology 56, 61-67.

Buffery, A. W. H. \& Gray, J. A. (1972). Sex differences in the development of perceptual and linguistic skills. In Gender Differences: Their Ontogeny and Significance (ed. C. Ounsted and D. C. Taylor), pp. 123-157. Churchill: London.

Butler, S. R. \& Norrsell, U. (1968). Vocalisation possibly initiated by the minor hemisphere. Narure 220, 793-794.

Cernacek, J. (1961). Contralateral motor irradiation-cerebral dominance. Archives of Neurology and Psychiarry 4, 165-172.

Corballis, M. C. \& Beale, I. L. (1976). The Psychology of Left and Right, Lawrence Erlbaum: Hillsdale, N.J.

Dimond, S. J. (1972). The Double-Brain. Churchill Livingstone: London \& Edinburgh.

Dimond, S. \& Beaumont, G. (1972). Processing in perceptual integration between and within the cerebral hemispheres. British Journal of Psychology 63, 509-514.

Dimond, S. J. \& Blizard, D. A. (1977). Evolution and Lateralisation of the Brain. New York Academy of Sciences: New York.

Fog, E. \& Fog. M. (1963). Cerebral inhibition examined by associated movements. In Minimal Cerebral Dysfunction (ed. R. MacKeith and M. Bax), pp. 52-57. Little Club Clinics in Developmental Medicine, No. 10. Heinemann: London.
Forgus, R. H. (1966). Perception. McGraw-Hill: New York.

Gazzaniga, M.S. \& Hillyard, S. A. (1971). Language and speech capacity of the right hemisphere. Neuropsychologia 9, 273-280.

Goff, W. R., Rosner, B. S. \& Altison, T. (1962). Distribution of cerebral somatosensory evoked responses in normal man. Electroencephalography and Clinical Neurophysiology 14, 697-713.

Harnard, S., Doty, R. W., Goldstein, L., Jaynes, J. \& Krauthamer, G. (eds.) (1977). Lateralization in the Nervous System. Academic Press: New York.

Harshman, R. A. \& Remington, R. (1975). Sex, language and the brain: 2. Adult sex differences in lateralization. Mimeographed.

Heap, M. \& Wyke, M. (1972). Learning of a unimanual skill in patients with brain lesions. Cortex $8,1-18$.

Hécaen, H. (1962). Clinical symptomatology in right and left hemisphere lesions. In Interhemispheric Relations and Cerebral Dominance (ed. V. B. Mountcastle), pp. 215-243. Johns Hopkins University Press: Baltimore.

Hécaen, H. \& Ajuriaguerra, J. de (1964). Left-handedness, Manual Superiority and Cerebral Dominance. Grune \& Stratton: New York.

Hécaen, H. \& Albert, M. L. (1978). Human Neuropsychology. Wiley: New York.

Hopf, H. C., Schlegel, H. J. \& Lowitzsch, K. (1974). Irradiation of voluntary activity to the contralateral site in movements of normal subjects and patients with central motor disturbances. European Neurology 12, 142-147.

Kinsbourne, M. (1971). The minor cerebral hemisphere. Archives of Neurology 25, 302-306.

Kinsbourne, M. (ed.) (1978). Asymmetrical Function of the Brain. Cambridge University Press: Cambridge.

Levy, J., Nebes, R. D. \& Sperry, R. W. (1971). Expressive language in the surgically separated minor hemisphere. Cortex 7, 49-58.

Milner, B. (1971). Interhemispheric differences and psychological process. British Medical Bulletin 27, 272-277.

Mountcastle, V. B. (ed.) (1962). Interhemispheric Relations and Cerebral Dominance. Johns Hopkins University Press: Baltimore.

Patton, H. D., Towe, A. L. \& Kennedy, T. T. (1962). Activation of pyramidal tract neurons by ipsilateral cutaneous stimuli. Journal of Neurophysiology 25, 501-514. 
Rosenzweig, M. R. (1951). Representation of the two ears at the auditory cortex. American Journal of Physiology 67, 147-158.

Schmitt, F. O. \& Worden, F. G. (eds.) (1974). The Neurosciences Third Study Program. MIT Press: Cambridge, Mass.

Schott, G. D. \& Wyke, M. A. (1977). Obligatory bimanual associated movements. Journal of the Neurological Sciences 33, 301-312.

Schott, G. D. \& Wyke, M. A. (1981). Congenital mirror movements. Journal of Neurology, Neurosurgery and Psychiatry 44, 586-599.

Sperry, R. W. (1962). Some general aspects of interhemispheric integration. In Interhemispheric Relations and Cerebral Dominance (ed. V. B. Mountcastle), pp. 5-19. Johns Hopkins University Press: Baltimore.
Terzulo, C. A. \& Adey, W. R. (1960). Sensorimotor activities. In Handbook of Physiology, Section I (Neurophysiology), vol. 2 (ed. J. Field, H. W. Magoun and V. Z. Hall), pp. 797-835. American Physiological Society: Washington, D.C

Teuber, H. L. (1974). Why two brains? In The Neurosciences Third Study Program (ed. F. O. Schmitt and F. G. Worden), pp. 71-74. MIT Press: Cambridge, Mass.

Wyke, M. (1969). Influence of direction in the rapidity of bilateral arm movements. Neuropsychologia 7, 189-194.

Zangwill, O. L. (1963). The cerebral localisation of psychological function. The Advancement of Science XX, 1963-64. 